\title{
Optical discrimination between malignant and benign breast lesions?
}

\author{
Giovanna Quarto $^{1}$, Antonio Pifferi ${ }^{1,2}$, Rinaldo Cubeddu ${ }^{1,2}$, Francesca Ieva ${ }^{3}$, Anna Maria Paganoni ${ }^{4}$, \\ Francesca Abbate ${ }^{5}$, Enrico Cassano ${ }^{5}$, Paola Taroni ${ }^{1}$ \\ ${ }^{1}$ Dipartimento di Fisica, Politecnico di Milano, Piazza L. da Vinci 32, 20133 Milano, Italy; ${ }^{2}$ Istituto \\ di Fotonica e Nanotecnologie, Consiglio Nazionale delle Ricerche, Piazza L. da Vinci 32, 20133 \\ Milano, Italy; ${ }^{3}$ Dipartimento di Matematica, Università degli Studi di Milano, Via C. Saldini 50 - \\ 20133 Milano, Italy; ${ }^{4}$ Dipartimento di Matematica, Politecnico di Milano, Piazza L. da Vinci 32, \\ 20133 Milano, Italy ${ }^{5}$ European Institute of Oncology, Breast Imaging Unit, Via G. Ripamonti, 435, \\ 20141 Milano, Italy
}

\begin{abstract}
Time domain multi-wavelength (635 to $1060 \mathrm{~nm}$ ) optical mammography was performed on 82 subjects with breast lesions (45 malignant and 38 benign lesions). A perturbative approach based on the high-order calculation of the pathlength of photons inside the lesion was applied to estimate differences between lesion and average healthy breast tissue in terms of: i) absorption properties, and ii) concentration of the major tissue constituents (oxy- and deoxy-hemoglobin, water, lipid and collagen). The absorption difference $\Delta \mu_{a}$ between lesion and healthy tissue is significantly different for malignant $v s$. benign lesions at all wavelengths. Logistic regression fitted to the absorption data identifies $975 \mathrm{~nm}$ as the key wavelength to discriminate malignant from benign lesions. When the difference in tissue composition between lesion and healthy tissue is considered, malignant lesions are characterized by significantly higher collagen content than benign lesions. Also the best model for the discrimination of malignant lesions obtained applying regression logistic to tissue composition is based only on collagen.
\end{abstract}

Keywords: Tissue diagnostics, optical mammography, time-resolved diffuse optical imaging, breast cancer.

\section{INTRODUCTION}

One in 8 women in the U.S. and 1 in 10 in most EU countries will develop breast cancer in their lifetime. Breast screening primarily relies on x-ray mammography, which has reduced sensitivity in patients with dense glandular breasts, including young women and may have limited specificity, leading to unnecessary treatment.

Optical mammography can operate at multiple wavelengths, combining imaging and spectroscopic information for lesion detection and characterization. In addition to its non-invasive nature, optical mammography has the capability to investigate dense breasts, typical of young women. The optical estimate of breast tissue composition might thus allow the discrimination between malignant and benign lesions. Most studies performed so far on the optical characterization of breast lesions have focused on the estimate of blood parameters, reporting increased blood content in breast cancers as compared to the surrounding healthy tissue. Only few studies quantified also of water and lipids, reporting a reduction in lipid content and an increase in water and blood compared to normal breast tissue.

Collagen is involved in the onset and progression of breast cancer and might prove useful for the detection and characterization of breast lesions. In the present study, we investigate the differences in absorption properties of malignant and benign lesions as compared to healthy tissue at 7 wavelengths in the range 635 to $1060 \mathrm{~nm}$, and estimate tissue composition in malignant and benign breast lesions, including also collagen among the tissue constituents that are considered.

\section{MATERIALS AND METHODS}

\subsection{Instrument set-up}

The instrument was designed to collect projection images in compressed breast geometry. Time-resolved transmittance measurements were performed at seven wavelengths (635, 685, 785, 905, 930, 975, $1060 \mathrm{~nm}$ ) using picosecond pulsed 
diode lasers and two PC-boards for time-correlated single photon counting. The compressed breast was raster-scanned continuously, moving the illumination fiber and collecting bundle in tandem and recording data every millimeter. Optical images were acquired from both breasts in cranio-caudal and medio-lateral oblique $\left(45^{\circ}\right)$ views.

\subsection{Patient study}

The Institutional Review Board at the European Institute of Oncology approved the clinical study. Written informed consent was obtained from all the participants. The study had twofold aim: i) the non-invasive assessment of breast density by optical means (not considered here), and ii) the optical characterization of malignant and benign lesions. A retrospective study was performed on 218 subjects, and optical images were analyzed comparing them to x-ray mammograms acquired in the same views. 82 subjects were further considered for the present analysis: 45 had malignant breast lesions (including 33 ductal invasive carcinomas) and 38 benign breast lesions (including 14 fibroadenomas).

\subsection{Data analysis}

Information on tissue composition and structure is obtained directly from time-resolved curves measured at 7 wavelengths. A spectrally constrained global fitting procedure, based on an analytical solution of the diffusion approximation (with the extrapolated boundary condition) for an infinite homogeneous slab, is applied. Free parameters of the fit are the concentrations of oxy- and deoxy-hemoglobin $\left(\mathrm{HbO}_{2}\right.$ and $\mathrm{Hb}$, respectively), water, lipids, and collagen concentrations, together with the scattering amplitude $a$ and power $b$.

The Beer law is then used to estimate the absorption coefficient at each wavelength from the concentrations of the main tissue constituents. By the knowledge of the parameters $a$ and $b$, the reduced scattering coefficient is modeled and obtained through a simple approximation to Mie theory: $\mu_{s}^{\prime}=a\left(\lambda / \lambda_{o}\right)^{-b}$, where we set $\lambda_{o}=600 \mathrm{~nm}$; in this way $a$ is the interpolated scattering coefficient $\mu_{s}^{\prime}\left(\lambda_{o}\right)$.

In order to optically characterize breast lesions, they were described as localized absorption perturbations, embedded in an otherwise homogeneous diffusive medium, and a perturbation model based on the high-order calculation of the pathlength of photons inside the lesion was applied. ${ }^{[1]}$ The perturbation model relies on the a priori knowledge of the volume and location of the inhomogeneity, which was always assumed as spherical, since only one dimension (i.e., the maximum diameter) was known, and located halfway between the injection and detection points, as no information on the depth was available.

The statistical significance of the difference between two groups (e.g., absorption properties of malignant vs benign lesions at $635 \mathrm{~nm}$ ) was assessed using the Mann-Whitney test at alpha $=0.05$. Significance was considered when $p<0.05$. The best regression logistic models to identify malignancy based on absorption properties or tissue composition were chosen via a stepwise variables selection minimizing the AIC (Akaike Information Criterion).

\section{RESULTS AND DISCUSSION}

The perturbative approach applied in the present study allows the estimate of absorption differences $\Delta \mu_{a}$ between diseased tissue (either a malignant or a benign breast lesion) and average tissue in the same breast. On average malignant lesions are characterized by higher $\Delta \mu_{a}$ values than benign lesions. The difference is particularly marked at the shortest wavelengths and at $975 \mathrm{~nm}$ (Table 1 and Fig. 1). The estimated $\Delta \mu_{a}$ values are spread over a broad range. In this initial stage, very different lesion types were included in each of the two groups. This certainly affects the results and further analysis is needed to determine if specific lesion types have characteristic properties. However, there is a significant $\Delta \mu_{a}$ difference $(p<0.05)$ between the two general categories of malignant and benign lesions at each of the 7 wavelengths.

Table 1. Median of the absorption differences $\Delta \mu_{a}$ for malignant and benign breast lesions

\begin{tabular}{l|ccccccc}
\hline Lesion type & $\mathbf{6 3 5} \mathbf{~ n m}$ & $\mathbf{6 8 5} \mathbf{~ n m}$ & $\mathbf{7 8 5} \mathbf{~ n m}$ & $\mathbf{9 0 5} \mathbf{~ n m}$ & $\mathbf{9 3 0} \mathbf{~ n m}$ & $\mathbf{9 7 5} \mathbf{~ n m}$ & $\mathbf{1 0 6 0} \mathbf{~ n m}$ \\
\hline Malignant & 0.093 & 0.061 & 0.060 & 0.057 & 0.035 & 0.108 & 0.042 \\
Benign & 0.060 & 0.034 & 0.027 & 0.041 & 0.034 & 0.054 & 0.033 \\
\hline
\end{tabular}



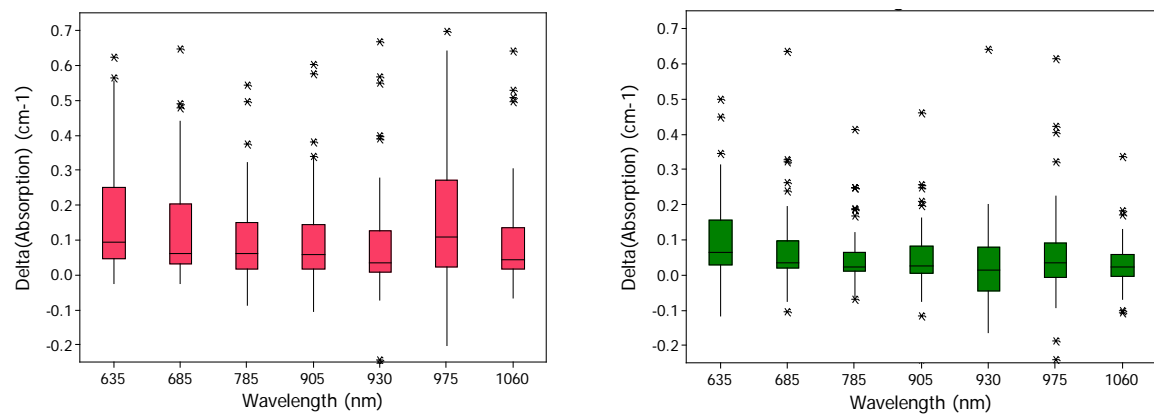

Figure 1. $\Delta \mu_{a}$ at 7 wavelengths $(635-1060 \mathrm{~nm})$ for malignant (red) a benign (green) breast lesions.

The best logistic regression model obtained fitting to the absorption differences estimated at all wavelengths turned out to depend only on $\Delta \mu_{a}$ at $975 \mathrm{~nm}$. It allows the discrimination of malignant lesions with sensitivity of 0.89 , but very low specificity $(0.28)$.

The differences $\Delta C_{i}$ in concentration of tissue constituents between breast lesions and healthy tissue were estimated relying on the Beer law (Fig. 2 and table 2). Only the difference in collagen content allows on average to distinguish malignant from benign lesions, with the former being characterized by significantly higher $(p=0.0018)$ collagen content than benign lesions with respect to healthy tissue.

Table 2. Median of the concentration differences $\Delta C_{i}$ for malignant and benign breast lesions

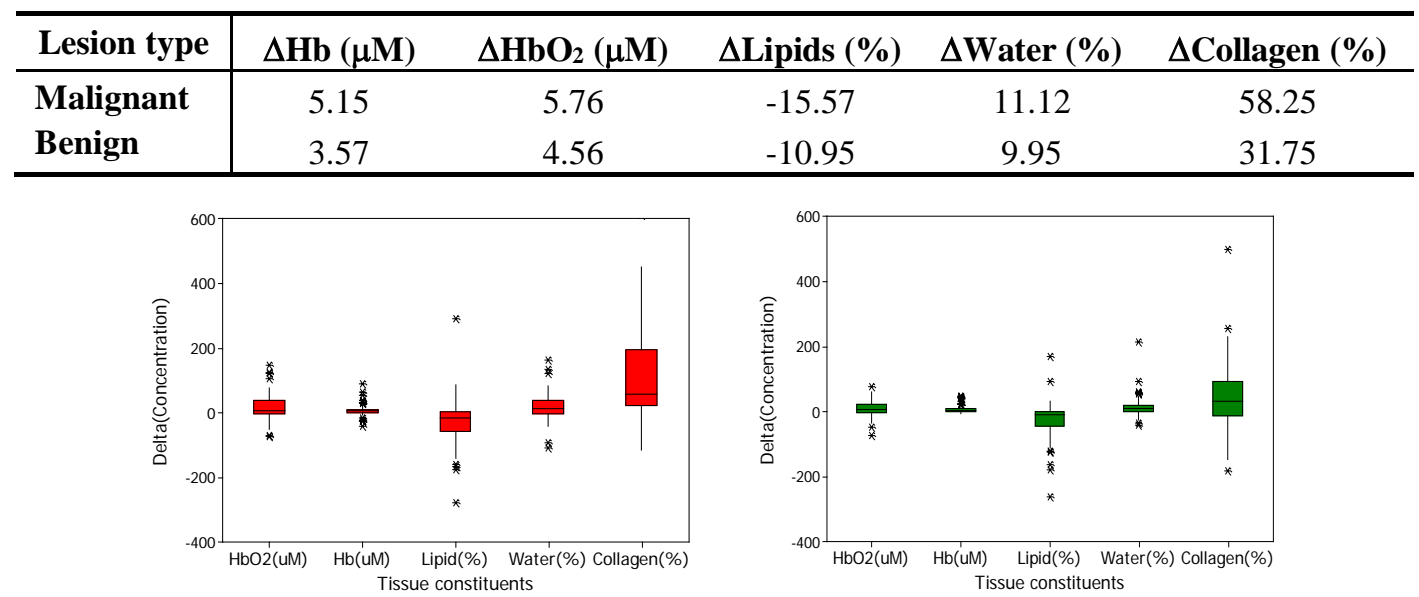

Figure 2. $\Delta C_{i}$ for malignant (red) a benign (green) breast lesions.

When concentration differences are considered, the best logistic regression model depends only on collagen content. It allowed the discrimination of malignant lesions with sensitivity of 0.88 , but the specificity was still low (0.32).

As mentioned previously, the two categories of malignant and benign lesions considered here include different types of lesions that may have very different composition and absorption properties. Moreover, different scattering properties can be envisaged (e.g., between liquid lesions, such as cysts, and solid ones, such as fibroadenomas). Taking them into account might improve the model. More in general, further analysis is being performed to achieve a better discrimination between malignant and benign lesions.

\section{REFERENCES}

[1] Sassaroli, A., Martelli, F. and Fantini, S., "Perturbation theory for the diffusion equation by use of the moments of the generalized temporal point-spread function. I. Theory," J. Opt. Soc. Am. A Opt. Image Sci. Vis. 23, 21052118 (2006). 